\title{
The Nature of the Mn Moment in Laves Phase Compounds: Evolution of the Magnetic Order in $\mathrm{Ho}_{1-x} \mathbf{Y}_{x} \mathbf{M n}_{2}$
}

\author{
Clemens Ritter ${ }^{1}$, Robert Cywinski ${ }^{2}$, and Sue H. Kilcoyne ${ }^{2, *}$ \\ 1 Institute Laue-Langevin, F-38042, Grenoble Cedex. \\ 2 J J Thomson Physical Laboratory, University of Reading, Reading RG6 2AF, UK \\ Z. Naturforsch. 50a, $191-198$ (1995); received September 26, 1994
}

Dedicated to Prof. Dr. W. Müller-Warmuth on the Occasion of his 65th Birthday

\begin{abstract}
Neutron powder diffraction has been used to study the evolution of long range magnetic order in the pseudobinary $\mathrm{C} 15$ Laves phase system $\mathrm{Ho}_{1-x} \mathrm{Y}_{x} \mathrm{Mn}_{2}$. Particular attention has been paid to the nature of the Mn moment. At Y-rich compositions $(x>0.9)$ an incommensurate antiferromagnetic structure, similar to that of $\mathrm{YMn}_{2}$ is observed. Transition to the ordered state, as in $\mathrm{YMn}_{2}$, is accompanied by a $5 \%$ expansion of the unit cell, identifying the Mn moments, of $2.7 \mu_{B}$, as intrinsic. The magnetic structure of compositions with $x<0.7$ resembles that of $\mathrm{HoMn}_{2}$ and $\mathrm{DyMn}_{2}$, with only one quarter of the chemically equivalent $\mathrm{Mn}$ sites possessing a moment of $0.6 \mu_{\mathrm{B}}$ induced by the local symmetry of the antiferromagnetically ordered Ho sublattice. Transition to the ordered state is not accompanied by a cell expansion. Between $x=0.7$ and $x=0.9$ there is no longe range magnetic order, nor is there an expansion of the unit cell, suggesting the total absence of either induced or intrinsic $\mathrm{Mn}$ moments. The results indicate that a critical $\mathrm{Mn}-\mathrm{Mn}$ near neighbour distance of $2.663 \AA$ is necessary to sustain an intrinsic Mn moment in these compounds.
\end{abstract}

Key words: $\mathrm{Ho}_{1-x} \mathrm{Y}_{x} \mathrm{Mn}_{2}$, Magnetic structure, Neutron diffraction, Laves phase.

\section{Introduction}

The magnetic ground state of manganese in the $\mathrm{C} 14$ and $\mathrm{C} 15$ Laves phase compounds $\mathrm{RMn}_{2}$, where $\mathrm{R}$ is a $4 \mathrm{f}$ metal or $\mathrm{Y}$, has been shown to depend sensitively on the $\mathrm{Mn}-\mathrm{Mn}$ near neighbour distance, $d_{\mathrm{Mn}-\mathrm{Mn}}$ [1]. At high temperatures $(T>100 \mathrm{~K})$ inelastic neutron scattering and $\mu \mathrm{SR}$ show the Mn magnetic moments to be in a spin fluctuating state [2]. With decreasing temperature those compounds with $d_{M n-M n}$ larger then $2.66 \AA$ develop an intrinsic moment at the $\mathrm{Mn}$ sites of approximately $2.8 \mu_{\mathrm{B}}$ [3]. The sudden appearance of this localised moment coincides with the onset of antiferromagnetic order and is accompanied by a marked increase in the volume of the unit cell. In the case of $\mathrm{YMn}_{2}$ the volume anomaly, $\Delta V / V$, is as large as $5 \%$ [4]. In contrast, for those compounds with $d_{\mathrm{Mn}-\mathrm{Mn}}<2.66 \AA$ only some of the Mn sites develop a moment [5]. In this case the Mn moment is induced by a strongly polarising magnetic environment arising from the magnetically ordered $4 \mathrm{f}$ sublattice. For example, in cubic $\mathrm{C} 15 \mathrm{DyMn}_{2}$ and $\mathrm{HoMn}_{2}$ one in four of the chemically equivalent $\mathrm{Mn}$ sites possess an in-

* Present address: ISIS, Rutherford Appleton Laboratory, Chilton, OX11 0QX, UK.

Reprint requests to Dr. C. Ritter. duced moment $[5,6]$. The differences in character of the intrinsic and induced moments are underlined by the absence of a volume anomaly associated with the appearance of the latter.

Neutron powder diffraction, magnetostriction measurements and $\mu \mathrm{SR}$ have already been used to map the evolution from induced to intrinsic $\mathrm{Mn}$ moment in the pseudobinary system $\mathrm{Dy}_{1-x} \mathrm{Y}_{x} \mathrm{Mn}_{2}$ [7-10]. It has been shown that at intermediate concentrations, $0.7<1-x<0.3$, there is no longer range magnetic order. However, a volume anomaly of $\Delta V /$ $V=1 \%$ is observed at the "spin-glass" transition ( $\left.T_{\mathrm{g}}=20 \mathrm{~K}\right)$, providing evidence for the persistence of an intrinsic moment, of the order of $1 \mu_{\mathrm{B}}$, throughout this magnetically disordered intermediate regime.

For $\mathrm{HoMn}_{2}$ the $\mathrm{Mn}-\mathrm{Mn}$ near neighbour distance is significantly smaller than that of $\mathrm{DyMn}_{2}$. In principle, therefore, the pseudobinary series $\mathrm{Ho}_{1-x} \mathrm{Y}_{x} \mathrm{Mn}_{2}$ affords the possibility of exploring the transition from induced to intrinsic moment in more detail than does $\mathrm{Dy}_{1-x} \mathrm{Y}_{x} \mathrm{Mn}_{2}$. We have used neutron powder diffraction to study the phase diagram of cubic C15 $\mathrm{Ho}_{1-x} \mathrm{Y}_{x} \mathrm{Mn}_{2}$ in order to elucidate the interplay of structure and $4 \mathrm{f}$ metal interactions in defining the magnetic groundstate of $\mathrm{Mn}$ in the $\mathrm{RMn}_{2}$ compounds. 


\section{Experimental}

Nine $15 \mathrm{gr}$ samples from the $\mathrm{Ho}_{1-x} \mathrm{Y}_{x} \mathrm{Mn}_{2}$ series in the range $0<x<1$ were prepared by argon arc melting appropriate quantities of the constituent elements of purity better than $99.9 \%$ (Johnson-Matthey plc). A slight excess $(5 \%)$ of $\mathrm{Ho}$ and $\mathrm{Y}$ over $\mathrm{Mn}$ was used to help suppress formation of the $\mathrm{R}_{6} \mathrm{Mn}_{23}$ phase. The resulting ingots were subsequently powdered.

The neutron diffraction experiments were performed using the D1B high intensity powder diffractometer at the Institut Laue-Langevin (ILL), Grenoble. D1B uses a $2.52 \AA$ monochromatic neutron beam and has a 400 element linear multidetector covering an angular range of $80^{\circ}$. The multidetector allows rapid data collection, typical diffraction patterns being accumulated in 10 minute intervals.

The powdered samples were contained in a vanadium can mounted in a standart ILL "orange" cryostat. Patterns were collected at temperatures between $2 \mathrm{~K}$ and $300 \mathrm{~K}$. The resulting diffraction patterns were analysed using programs developed at the ILL. Individual Bragg peak intensities were determined using the ABFFIT programs, while full structural and magnetic profile refinement was carried out using a modified Rietveld method, FULLPROF [11, 12].

\section{Results}

The thermal evolution of the neutron diffraction patterns from four selected $\mathrm{Ho}_{1-x} \mathrm{Y}_{x} \mathrm{Mn}_{2}$ samples is shown in Figures 1-4. These patterns are characteristic of those obtained in four principle regions of the $\mathrm{Ho}_{1-x} \mathrm{Y}_{x} \mathrm{Mn}_{2}$ magnetic phase diagram, which is shown in Figure 5. These four regions will be discussed in turn below.

\section{$0<x<0.35$}

The thermogram in Fig. 1 is typical of those obtained from the Ho-rich samples with $x=0.1,0.2$ and 0.3 . Initially antiferromagnetic Bragg peaks, associated with the AF2 structure, develop on cooling. On further cooling evidence for spin canting is provided by the appearance of a ferromagnetic $(\mathrm{F})$ contribution at the nuclear Bragg peaks. The low temperature spectra could be fitted using the spin canted $(\mathrm{AF} 2+\mathrm{F})$

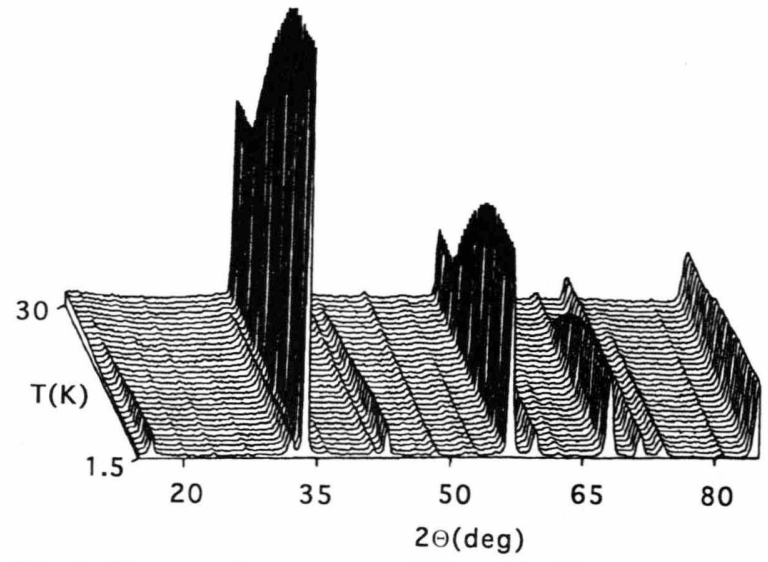

Fig. 1. Neutron thermogram of $\mathrm{Ho}_{0.9} \mathrm{Y}_{0.1} \mathrm{Mn}_{2}$.

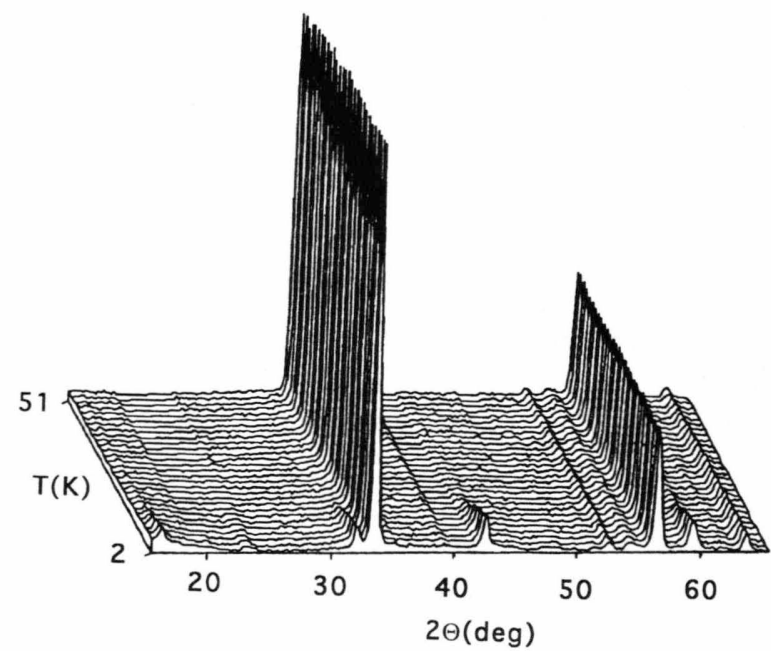

Fig. 2. Neutron thermogram of $\mathrm{Ho}_{0.5} \mathrm{Y}_{0.5} \mathrm{Mn}_{2}$.

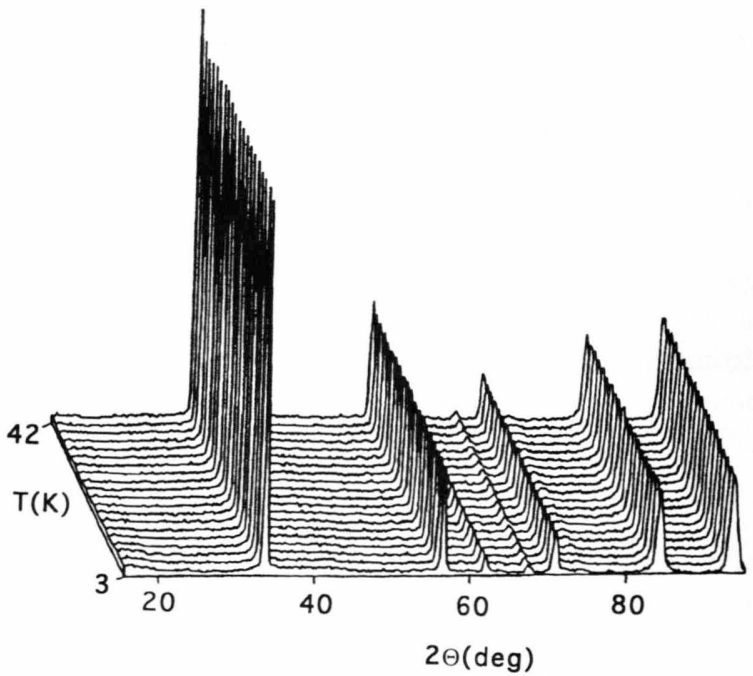

Fig. 3. Neutron thermogram of $\mathrm{Ho}_{0.3} \mathrm{Y}_{0.7} \mathrm{Mn}_{2}$. 


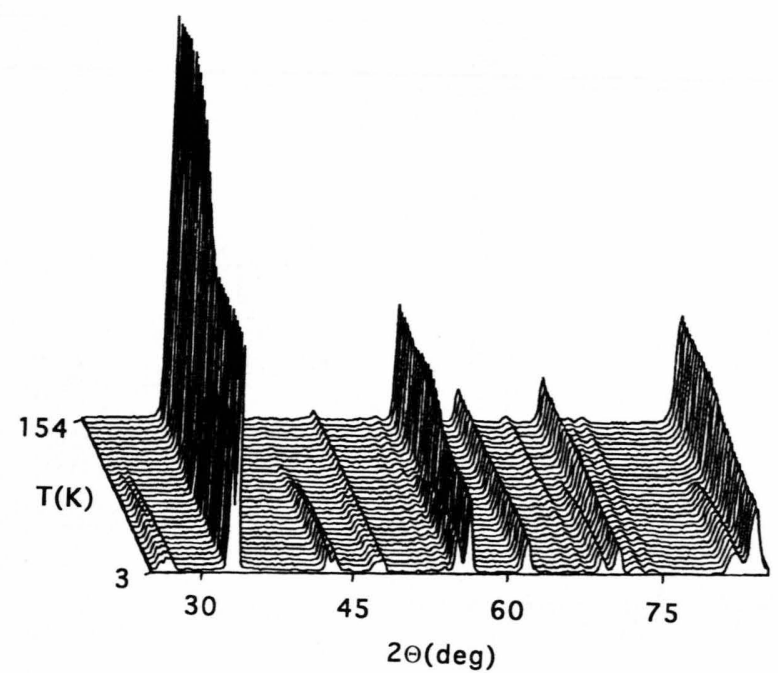

Fig. 4. Neutron thermogram of $\mathrm{Ho}_{0.05} \mathrm{Y}_{0.95} \mathrm{Mn}_{2}$.

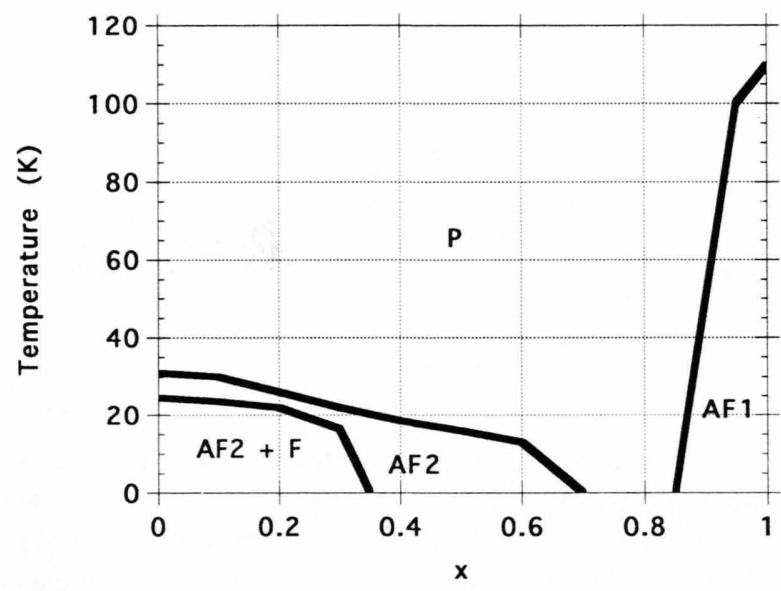

Fig. 5. The magnetic phase diagram of $\mathrm{Ho}_{1-x} \mathrm{Y}_{x} \mathrm{Mn}_{2}$. $\mathrm{P}$ stands for paramagnetic, AF1 and AF2 for antiferromagnetic order of first and second type for a cubic fec lattice and $\mathrm{F}$ stands for ferromagnetism.

structure reported previously for the pure $\mathrm{DyMn}_{2}$ and $\mathrm{HoMn}_{2}[5,6]$ compounds and illustrated in Figure 6. Increasing the substitution of nonmagnetic $\mathrm{Y}$ leads to a decrease in the magnitude of the ferromagnetic component (and hence in the spin canting) whilst the antiferromagnetic component is little changed (Table 1). As it is the antiferromagnetic component of the $4 \mathrm{f}$-metal spin structure that is responsible for the exchange induced magnetic moment at one

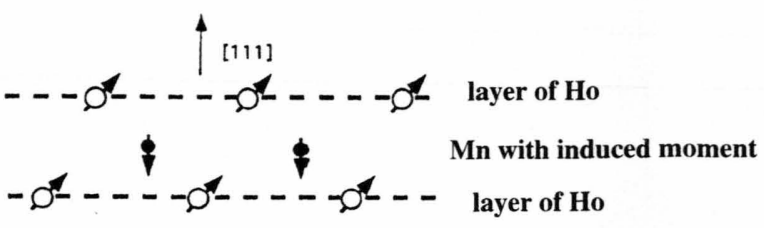

- $\quad \bullet \quad$ • $\quad$ nonmagnetic Mn

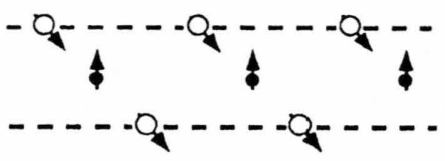

Fig. 6. The magnetic structure of $\mathrm{Ho}_{1-x} \mathrm{Y}_{x} \mathrm{Mn}_{2}$ with $0 \leq x<0.65$. For more details see [5] and [8].

Table 1. Refined values of the magnetic moments in $\mathrm{Ho}_{1-x} \mathrm{Y}_{x} \mathrm{Mn}_{2}$ tabulated as function of $1-x . F$ and $A F$ stand for ferro- and antiferromagnetic component of the rare earth sublattice. $F_{\text {corr }}$ and $A F_{\text {corr }}$ are values corrected for the actual Ho concentration and give the values per Ho atom. Mn stands for Manganese moment. All values are in units of $\mu_{B}$.

\begin{tabular}{llllllll}
\hline $1-x$ & $F$ & & $F_{\text {corr }}$ & \multicolumn{1}{l}{$A F$} & \multicolumn{2}{l}{$A F_{\text {corr }}$} & \multicolumn{2}{l}{ Mn } \\
\hline 0.3 & - & - & - & & - & - & \\
0.4 & - & - & 1.17 & $(6)$ & $2.93(15)$ & 0.2 & $(1)$ \\
0.5 & - & - & 2.25 & $(5)$ & $4.50(10)$ & $0.25(10)$ \\
0.6 & - & & - & $3.72(5)$ & $6.20(8)$ & 0.55 & $(7)$ \\
0.7 & $2.89(9)$ & $4.13(13)$ & $3.82(6)$ & $5.45(9)$ & $0.65(6)$ \\
0.8 & $4.93(13)$ & $6.16(16)$ & $3.62(15)$ & $4.53(19)$ & $0.63(10)$ \\
0.9 & $6.07(16)$ & $6.74(18)$ & $3.00(18)$ & $3.33(20)$ & $0.52(13)$ \\
1.0 & $7.03(20)$ & $7.03(20)$ & $3.46(21)$ & $3.46(21)$ & $0.62(20)$ \\
\hline
\end{tabular}

in four of the Mn sites, the magnitude of the induced Mn moment remains constant at $0.6 \mu_{\mathrm{B}}$. "Curie" and Néel temperatures have been determined from the variation of the magnetic Bragg intensities with temperature. Both decrease with increasing Y substitution. Table 1 summarises the principal magnetic parameters obtained from refinement of the magnetic structures, together with the previously reported values for $\mathrm{HoMn}_{2}$.

Sequential refinement of the lattice parameter was performed for all three samples in this concentration regime. Although the thermal expansion coefficient (TEC) above $T_{\mathrm{N}}$ is moderately enhanced (TEC $\left.=27 \times 10^{-6} \mathrm{~K}^{-1}\right)$ indicative of spin fluctuating $3 \mathrm{~d}$ moments, there is no sign of a volume anomaly at $T_{\mathrm{N}}$ for any of the samples. 
Intensity (counts)

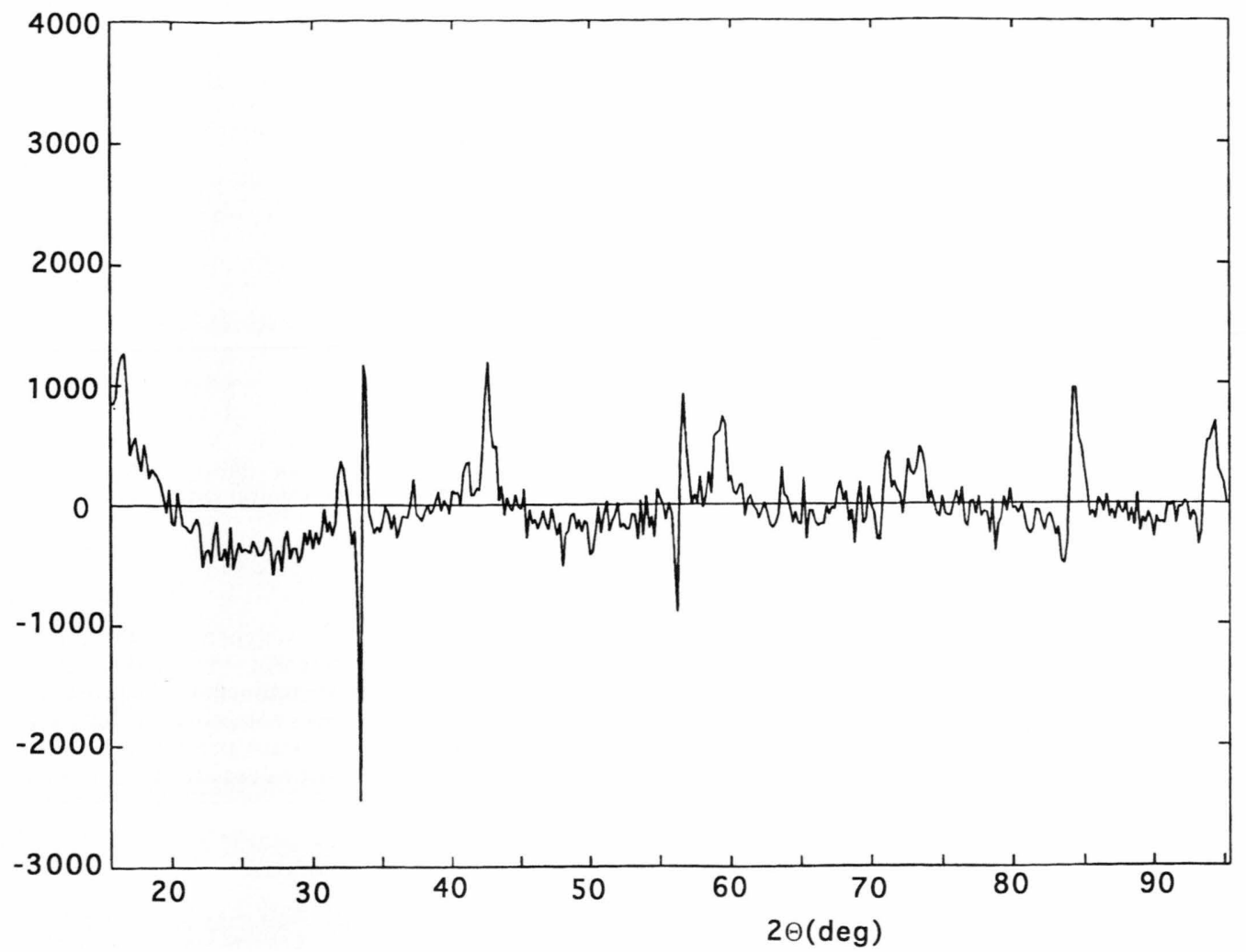

Fig. 7. The $30 \mathrm{~K}-2 \mathrm{~K}$ difference pattern of $\mathrm{Ho}_{0.4} \mathrm{Y}_{0.6} \mathrm{Mn}_{2}$.

\section{$0.35<x<0.65$}

This range of the magnetic phase diagram is characterised by the disappearance of the ferromagnetic component of the spin structure of the rare earth sublattice. The antiferromagnetic coupling persists, however, as can be seen in the thermogram of $\mathrm{Ho}_{0.5} \mathrm{Y}_{0.5} \mathrm{Mn}_{2}$ (Figure 2). The values of the magnetic moments and ordering temperatures extracted from refinement of the diffraction patterns are listed in Table 1.

$\mathrm{Ho}_{0.6} \mathrm{Y}_{0.4} \mathrm{Mn}_{2}$ sees an AF2 coupling comparable to that of the Ho-rich samples discussed above, and consequently the induced $\mathrm{Mn}$ moment on every fourth site remains constant at $0.6 \mu_{\mathrm{B}}$. However, further dilution by $\mathrm{Y}$ decreases the $4 \mathrm{f}$-metal exchange coupling and hence the magnitude of the Mn moments. Figure 7 shows the difference between $\mathrm{Ho}_{0.4} \mathrm{Y}_{0.6} \mathrm{Mn}_{2}$ spectra collected at $2 \mathrm{~K}$ and $30 \mathrm{~K}$. While AF2 antifer- romagnetic Bragg peaks are still evident, the difference spectrum is dominated by a modulated background arising from massive magnetic short range $4 \mathrm{f}$ spin correlations associated with the AF2 phase. Statistics are not sufficient to analyse this magnetic short range order quantitatively doing a Fourier transform analysis. As a rough estimate an upper limit for the correlation length of $30 \AA$ can be determined using the Scherrer formula for peak broadening [13].

Again, in this concentration range, sequential refinement of the structural parameters shows no volume anomaly at $T_{\mathrm{N}}$. A TEC of $29 \times 10^{-6} \mathrm{~K}^{-1}$ is obtained.

\section{$0.65<x<0.85$}

The thermogram of $\mathrm{Ho}_{0.3} \mathrm{Y}_{0.7} \mathrm{Mn}_{2}$ (Fig. 3) shows no evidence of long range magnetic order down to 


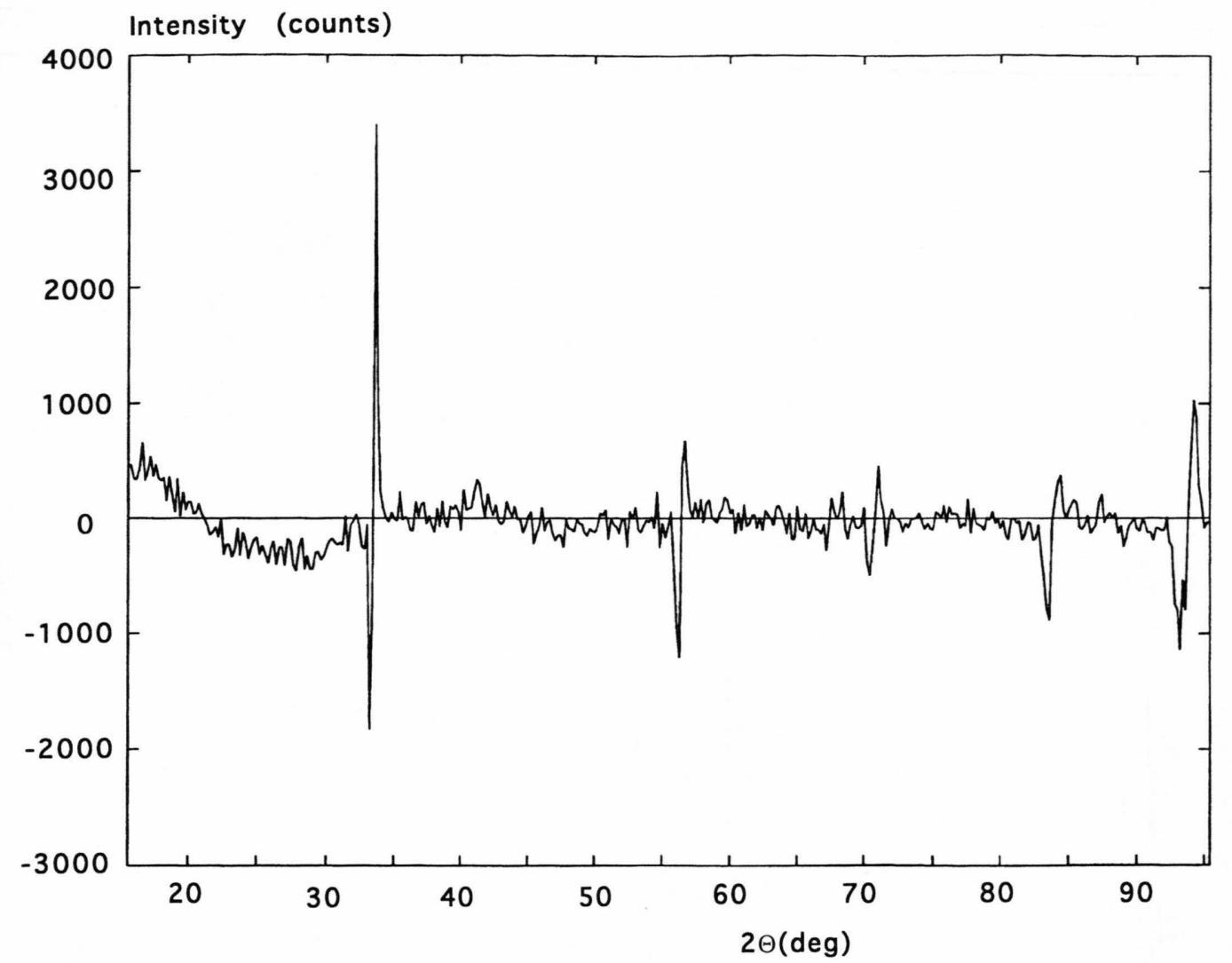

Fig. 8. The $30 \mathrm{~K}-5 \mathrm{~K}$ difference pattern of $\mathrm{Ho}_{0.3} \mathrm{Y}_{0.7} \mathrm{Mn}_{2}$.

$2 \mathrm{~K}$. The difference plot, $5 \mathrm{~K}-30 \mathrm{~K}$, for this compound (Fig. 8) provides evidence of persisting AF2 correlations, with a correlation length of again less than $30 \AA$. The antiferromagnetic Dy-Dy correlations are therefore of considerably shorter range than those observed in the "nonmagnetic" state at intermediate concentrations of the $\mathrm{Dy}_{1-x} \mathrm{Y}_{x} \mathrm{Mn}_{2}$ series where they amounted to about $100 \AA$ [8]. Also in contrast to the $\mathrm{Dy}_{1-x} \mathrm{Y}_{x} \mathrm{Mn}_{2}$ compounds, sequential refinement of the $\mathrm{Ho}_{0.3} \mathrm{Y}_{0.7} \mathrm{Mn}_{2}$ spectra shows no volume anomaly at any temperature.

\section{$0.85<x<1$}

Figure 4 shows the neutron thermogram obtained from the $\mathrm{Ho}_{0.05} \mathrm{Y}_{0.95} \mathrm{Mn}_{2}$ sample. The principal features of the thermogram are similar to those found in most dilute rare earth substituted $\mathrm{YMn}_{2}$ compounds.
There is a transition to antiferromagnetic order close to $T_{\mathrm{N}}=100 \mathrm{~K}$, which is accompanied by a massive thermal expansion anomaly. In this compound, however, only $34 \%$ of the sample transforms to the expanded antiferromagnetic phase, the remaining volume fraction staying paramagnetic. This results in an apparent decrease of the peak intensity of the nuclear Bragg peaks as the intensity is distributed between split nuclear peaks below $T_{\mathrm{N}}$.

In Fig. 9 the spectrum obtained from $\mathrm{Ho}_{0.05}$ $\mathrm{Y}_{0.95} \mathrm{Mn}_{2}$ at $2 \mathrm{~K}$ is shown. The split nuclear peaks reveal the coexistence of the expanded and nonexpanded phases. The antiferromagnetic Bragg peaks are worthy of closer inspection: at low angles where the resolution of the D1B diffractometer is optimal, one can clearly distinguish a splitting of these peaks. The first three magnetic peaks at $2 \Theta=31^{\circ}, 42^{\circ}$ and $47^{\circ}$ can be indexed, using the AF1 model proposed for $\mathrm{YMn}_{2}$ [14], as 110, 201 and 112. For $\mathrm{YMn}_{2}$ a propa- 


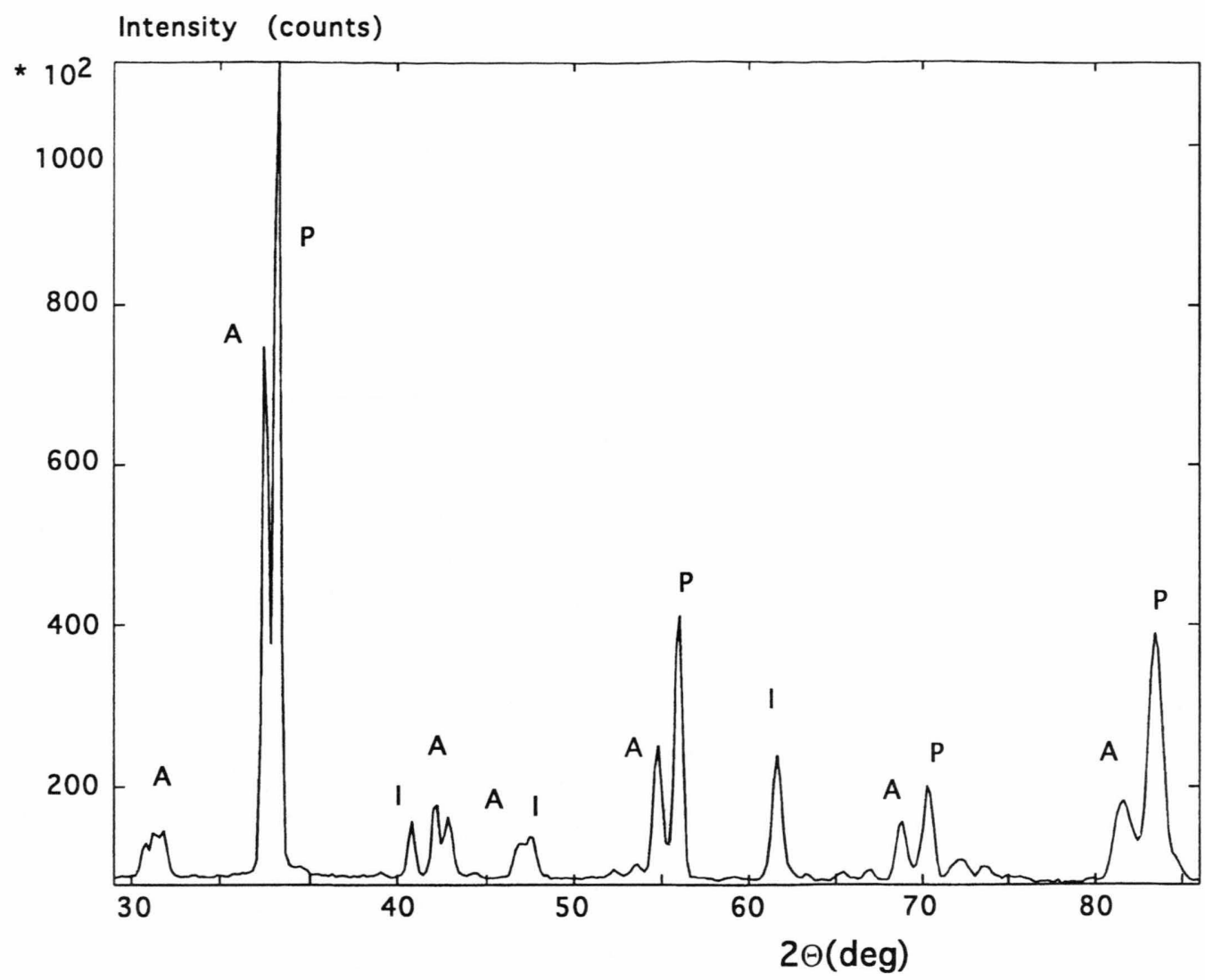

Fig. 9. The $2 \mathrm{~K}$ neutron diffraction pattern of $\mathrm{Ho}_{0.05} \mathrm{Y}_{0.95} \mathrm{Mn}_{2}$. A stands for antiferromagnetic phase, $\mathrm{P}$ for paramagnetic phase and I for impurity phase.

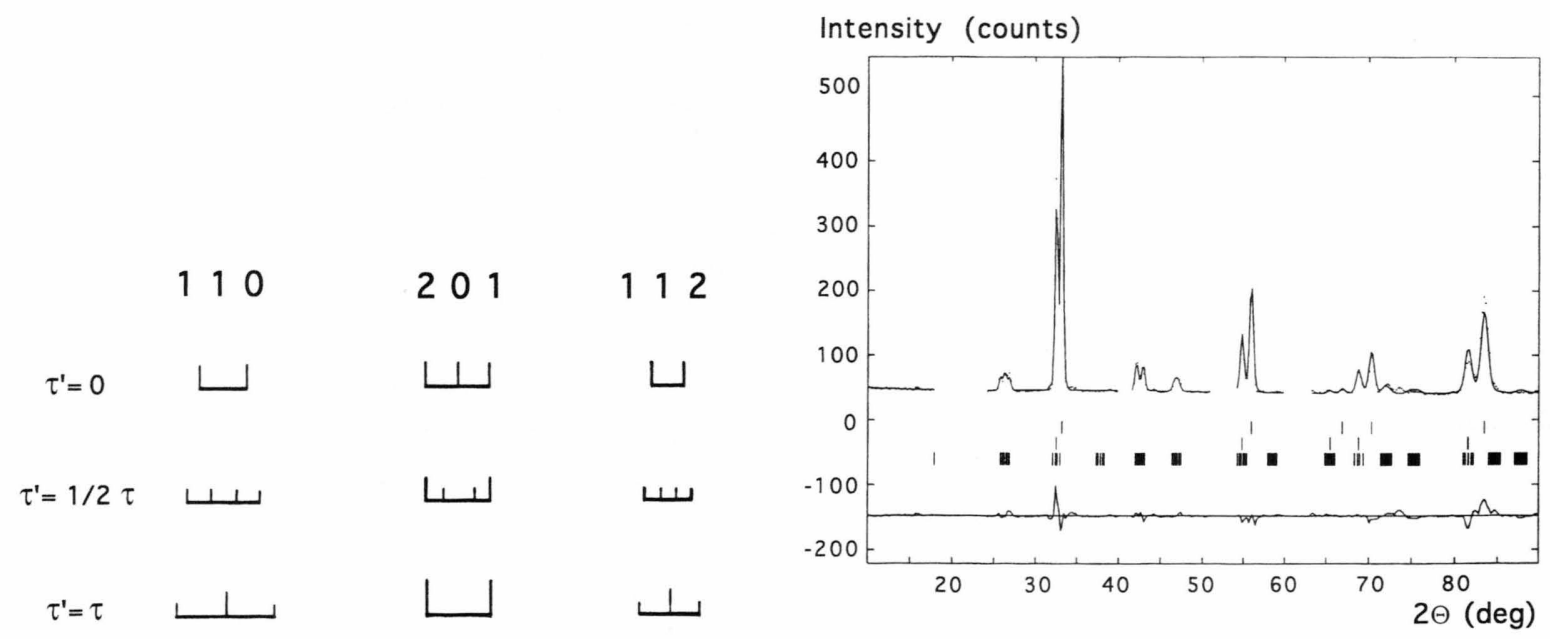

Fig. 10. A schematic presentation of the influence of the second component $\tau^{\prime}$ of the propagation vector $\left[\tau \tau^{\prime} 1\right]$ on the splitting of the first 3 antiferromagnetic reflections in $\mathrm{Ho}_{0.05} \mathrm{Y}_{0.95} \mathrm{Mn}_{2}$.

Fig. 11. The observed, calculated and difference powder neutron diffraction profils of $\mathrm{Ho}_{0.05} \mathrm{Y}_{0.95} \mathrm{Mn}_{2}$ at $2 \mathrm{~K}$ using a propagationvector $\left[\tau \tau^{\prime} 1\right]$. 
gation vector $\left[\tau \tau^{\prime} 1\right]$, with $\tau=0.018$ and $\tau^{\prime}=0.003$ has been determined from high resolution powder diffraction measurements [15]. In $\mathrm{Ho}_{0.05} \mathrm{Y}_{0.95} \mathrm{Mn}_{2}$ the observed splitting of the magnetic peaks, and hence the magnitude of the $\tau$-vectors, have significantly increased.

In Fig. 10 we explain schematically the influence of the two incommensurate components of the propagation vector on the splitting of the 110, 201 and 112 reflections: in particular note that when $\tau=\tau^{\prime}$ three peaks are observed at the 110 position while two peaks are found at 201. Using a modified Rietveld program which allows the refinement of incommensurate magnetic structures we obtain for $\mathrm{Ho}_{0.05} \mathrm{Y}_{0.95} \mathrm{Mn}_{2}$ at $2 \mathrm{~K}$ the propagation vector [ $\left.\tau \tau^{\prime} 1\right]$, with $\tau=0.024$ and $\tau^{\prime}=0.015$ (Figure 11).

It can be seen in Fig. 4 that the shape of the 110 peak evolves with temperature. A sequential Rietveld refinement of the temperature dependent magnetic structure of $\mathrm{Ho}_{0.05} \mathrm{Y}_{0.95} \mathrm{Mn}_{2}$ provides the components of the propagation vector shown in Figure 12. While $\tau$ remains almost constant at $0.023(1), \tau^{\prime}$ decreases significantly from 0.015 at $2 \mathrm{~K}$ to 0.0065 at $83 \mathrm{~K}$.

The value of the Mn moment in the expanded antiferromagnetic phase is $2.6(1) \mu_{\mathrm{B}}$ at $2 \mathrm{~K}$ and $2.75(15) \mu_{\mathrm{B}}$ at $83 \mathrm{~K}$, at which temperature only $12 \%$ of the sample volume is still in the expanded phase.

Refining the cubic lattice parameters a volume expansion of $\Delta V / V=5.8 \%$ is observed on cooling into the antiferromagnetic phase. This identifies the Mn moment as intrinsic. Correspondingly, in the paramagnetic phase, we find TEC $=53 \times 10^{-6} \mathrm{~K}^{-1}$. This

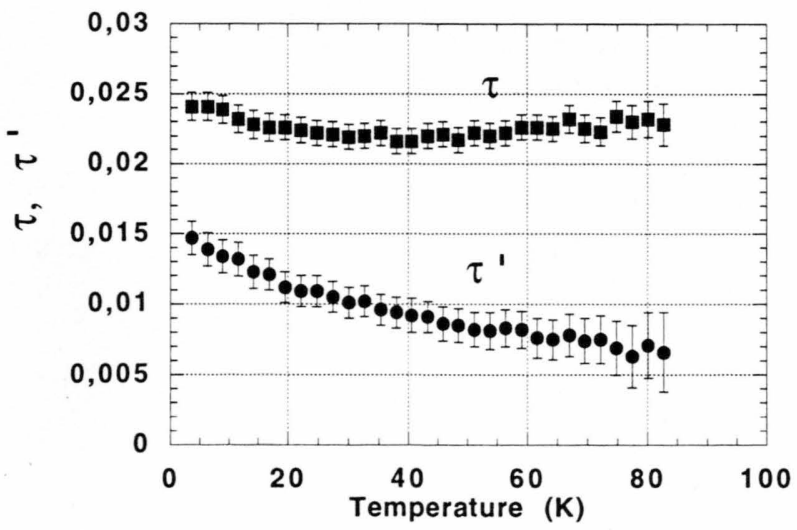

Fig. 12. Temperature dependence of the components $\tau$ and $\tau^{*}$ of the incommensurate propagation vector $\varkappa=\left[\tau \tau^{*} 1\right]$ of $\mathrm{Ho}_{0.05} \mathrm{Y}_{0.95} \mathrm{Mn}_{2}$.
Table 2. Results of the refinement for the Y-richest phases. $a_{0}$ para and $a_{0}$ anti stand for the cubic lattice parameters in the para- and antiferromagnetic part of the sample volume at $2 \mathrm{~K}$.

\begin{tabular}{lll}
\hline & $\mathrm{Ho}_{0.1} \mathrm{Y}_{0.9} \mathrm{Mn}_{2}$ & $\mathrm{Ho}_{0.05} \mathrm{Y}_{0.95} \mathrm{Mn}_{2}$ \\
\hline$\mu_{\mathrm{Mn}}$ & $2.65(35) \mu_{\mathrm{B}}$ & $2.6(1) \mu_{\mathrm{B}}$ \\
$\Delta V / V$ & $4.7 \%$ & $5.8 \%$ \\
$a_{0}$ para & $7.535(95 \%)$ & $7.543(66 \%)$ \\
$a_{0}$ anti & $7.652(5 \%)$ & $7.686(34 \%)$ \\
TEC & $40 \times 10^{-6} \mathrm{~K}^{-1}$ & $53 \times 10^{-6} \mathrm{~K}^{-1}$ \\
\hline
\end{tabular}

value, like that of the parent compound $\mathrm{YMn}_{2}$ is anomalously high, and is consistent with a spin fluctuating state above $T_{\mathrm{N}}$.

For the $\mathrm{Ho}_{0.1} \mathrm{Y}_{0.9} \mathrm{Mn}_{2}$ sample only a transformation to the expanded antiferromagnetic state is observed at $T_{\mathrm{N}}=60 \mathrm{~K}$. The magnetic structure is little changed from that of $\mathrm{Ho}_{0.05} \mathrm{Y}_{0.95} \mathrm{Mn}_{2}$, with the $\mathrm{Mn}$ moment refining to $2.65(15) \mu_{\mathrm{B}}$. The volume anomaly at $T_{\mathrm{N}}$ is $\Delta V / V=4.7 \%$ which is still very large and comparable to that of pure $\mathrm{YMn}_{2}$. The principal results obtained from the refinement of the two Y-rich samples are summarized in Table 2. It should be noted that there is no evidence of the participation of $\mathrm{Ho}$ moments in the observed AF1 phase.

\section{Discussion and Conclusions}

The pseudobinary C15 Laves phase system $\mathrm{Ho}_{1-x} \mathrm{Y}_{x} \mathrm{Mn}_{2}$ reveals a magnetic phase diagram which embraces a transition from a large $\left(\mu_{\mathrm{Mn}_{\mathrm{n}}}=2.7 \mu_{\mathrm{B}}\right)$ intrinsic localised $\mathrm{Mn}$ moment at high $\mathrm{Y}$ concentrations $(x>0.9)$ to a small $\left(\mu_{\mathrm{Mn}}=0.6 \mu_{\mathrm{B}}\right)$ induced $\mathrm{Mn}$ moment at one in four of the Mn sites at intermediate and high Ho concentrations $(x<0.7)$.

The transition between these two extremes does not proceed via a broad intermediate state characterised by disordered intrinsic $\mathrm{Mn}$ moments, as observed in the case of the related $\mathrm{Dy}_{1-x} \mathrm{Y}_{x} \mathrm{Mn}_{2}$ system [7]. Instead $\mathrm{Ho}_{1-x} \mathrm{Y}_{x} \mathrm{Mn}_{2}$ shows a relatively narrow disordered region $(0.7<x<0.9)$ in which there is no evidence for either intrinsic or induced moments at the Mn sites.

The apparent absence of Mn moments in this disordered region enabled us to determine accurately a critical $\mathrm{Mn}-\mathrm{Mn}$ distance above which an intrinsic Mn moment can be formed. We obtain $d_{\mathrm{c}}=2.663 \AA$, in very close agreement with the value extracted from our study of the $\mathrm{Dy}_{1-x} \mathrm{Y}_{x} \mathrm{Mn}_{2}$ phase diagram [8]. 
The existence of long range AF2-type magnetic order of the rate earth sublattice down to $\mathrm{Ho}_{0.04} \mathrm{Y}_{0.6} \mathrm{Mn}_{2}$ gives a strong indication of the important role R-Mn interactions play in the case of the $\mathrm{Dy}_{1-x} \mathrm{Y}_{x} \mathrm{Mn}_{2}$ series [7, 8]. There, in the presence of a small intrinsic moment on $\mathrm{Mn}$, only short range order is preserved on the Dy sublattice for $x>0.3$.

Although intrinsic moments cannot form for $d_{\mathrm{Mn}-\mathrm{Mn}}<d_{\mathrm{c}}$, the large TEC found in the paramagnetic phase at all compositions indicates presence of a spin fluctuating Mn moment, possibly close to localisation, to well below the critical distance.

The magnetic structure of the $\mathrm{YMn}_{2}$ samples with dilute Ho substitution is rather enigmatic: for dilute $\mathrm{Tb}$ and Dy substitution a commensurate AF1 phase is observed [7, 16].

It has been suggested that in pure $\mathrm{YMn}_{2}$ the magnetic frustation resulting from the superposition of

[1] M. Shiga, Physica B 149, 293 (1988).

[2] B. D. Rainford, S. Dakin, R. Cywinski, J. Magn. Magn. Mater. 104-107, 1257 (1992).

[3] B. Ouladdiaf, Thesis Grenoble 1986.

[4] Y. Nakamura, J. Magn. Magn. Mater. 31-34, 829 (1983).

[5] C. Ritter, S. H. Kilcoyne, and R. Cywinski, J. Phys: Condens. Matter 3, 727 (1991).

[6] C. Ritter, S. Mondal, S. H. Kilcoyne, and R. Cywinski, J. Phys: Condens Matter 4, 1559 (1992).

[7] C. Ritter, S. Mondal, S. H. Kilcoyne, R. Cywinski, and B. D. Rainford, J. Magn. Magn. Mater. 104-107, 1427 (1992).

[8] C. Ritter, R. Cywinski, S. H. Kilcoyne, S. Mondal, and B. D. Rainford, Phys. Rev. B 50, 9894 (1994).

[9] C. Ritter, C. Marquina, and M. R. Ibarra, sub. J. Magn. Magn. Mater. antiferromagnetic order upon a tetrahedral Mn-site topology gives rise to both a tetragonal distortion of the unit cell and an incommensurate magnetic structure $[15,17]$. For $\mathrm{Tb}$ and Dy substitution in $\mathrm{YMn}_{2}$ it appears that for these substituents at least, the magnetic frustration can be relieved without recourse to incommensurability: diffraction studies of these compounds have not yet been performed at sufficiently high resolution to assess the role of any tetragonal distortion.

We might speculate that in the case of Ho substitution the tetragonal distortion of the lattice below $T_{\mathrm{N}}$ is suppressed, leaving incommensurability as the only route by which the magnetic frustration can be relieved. Such speculation can only be resolved with further, high resolution, neutron diffraction measurements.

[10] M. Telling, R. Cywinski, S. H. Kilcoyne, and C. Ritter, to be published.

[11] A. Filhol, J. Y. Blanc, A. Anoniadis, and J. Berruyer, Institut Laue-Langevin Report 88FI05T (1988).

[12] J. Rodriguez-Carvajal, M. Anne, and J. Pannetier, Institut Laue-Langevin Report 87RO114T (1987).

[13] C. Legrand, Bull. Soc. Fr. Cer. 95, 101 (1972 ).

[14] T. Freltoft, B. Boni, G. Shirane, and K. Motoya, Phys. Rev. B 37, 3454 (1988).

[15] R. Cywinski, S. H. Kilcoyne, and C. A. Scott, J. Phys: Condens. Matter 3, 6473 (1991).

[16] R. Ballou, J. Deportes, R. Lemaire, R. Rouault, and J. L. Soubeyroux, J. Magn. Magn. Mater. 90-91, 559 (1990).

[17] R. Ballou, J. Deportes, R. Lemaire, Y. Nakamura, and B. Ouladdiaf, J. Magn. Magn. Mater. 70, 129 (1987). 